\title{
Parametric Modeling of Radiation Patterns and Scattering Parameters of Antennas
}

\author{
Ngoy Mutonkole, Elizabeth Rita Samuel, Dirk I. L. de Villiers, Senior Member, IEEE, and Tom Dhaene, Senior \\ Member, IEEE
}

\begin{abstract}
This paper describes a data-driven method to model the radiation patterns (over a large angular region) and scattering parameters of antennas as a function of the geometry of the antenna. The radiation pattern model consists of a linear combination of characteristic basis function patterns (CBFPs), where the expansion coefficients of the CBFPs are functions of geometrical features of the antenna. Scattering parameters are modeled by means of parameterized state-space matrices. The obtained models are quick to evaluate and are thus suitable for design activities where multiple simulations are required. The proposed method is validated through illustrative examples.
\end{abstract}

Index Terms-Antenna radiation patterns, interpolation, modeling, state-space methods.

\section{INTRODUCTION}

$\mathbf{M}$ ODERN antenna design procedures often involve activities such as design space exploration, optimization and sensitivity analysis. These normally require multiple fullwave and/or asymptotic electromagnetic (EM) analyses of the antenna for different geometric parameter values, and are expensive due to the typically large computational cost per simulation.

Model based parameter estimation (MBPE) is a modeling technique described by Miller in [1]-[4], and can be summarized as follows: a few points are used to interpolate a given response in time/frequency or in terms of angular dependence, using an underlying function that is based on the physics of the problem at hand (e.g., the sinc function for the radiation pattern of a reflector antenna in [3]).

Following the MBPE approach, techniques to model radiation patterns of simple feeds such as dipoles have been described in [5], [6]. These models interpolate the pattern in both frequency and a wide angular region $\Omega=(\theta, \phi)$ in the spherical coordinate system. The frequency dependence is expressed as a Padé rational function, while the angular variation of the pattern is expressed as polynomials in [5] or in terms of spherical harmonics [6]. Another pattern modeling method was proposed in [7] where the angular variation is also expressed as spherical harmonics while the frequency dependence is described using Slepian mode expansion. These

This work was financially supported in part by the South African Research Chair Initiative of the Department of Science and Technology.

Ngoy Mutonkole and Dirk I. L. de Villiers are with the Department of Electrical and Electronic Engineering, Stellenbosch University, Stellenbosch 7600, South Africa (e-mail: ngoymutonx@gmail.com,ddv@sun.ac.za).

Elizabeth Rita Samuel and Tom Dhaene are with the Department of Information Technology (INTEC), Ghent University - iMinds, Gaston Crommenlaan 8 Bus 201, B-9050, Gent, Belgium (e-mail: \{ elizabeth.ritasamuel, tom.dhaene\}@ugent.be). techniques may not be suitable to model the radiation pattern of electrically large antennas with rapidly varying radiation patterns as a function of $\Omega$ due to the poor interpolatory behavior (oscillations between exact-fit values) of high order polynomial representations of the pattern or due to the possibly large number of expansion coefficients that may be required in the case of the method in [6], [7]. Furthermore, the methods in [1]-[7] are not immediately suitable for design activities as the pattern models are not parameterized with respect to the antenna's physical dimensions (i.e., the design parameters). This limitation is addressed in this paper, where a model of the full radiation pattern as a function of varying antenna geometry is proposed.

A method to interpolate radiation patterns was recently proposed in [8], where the variation of the physical problem was captured by imposing a function such as a complex exponential or orthogonal polynomials. The method in [8] is based on a linear least-squares regression technique and is strongly dependent upon the choice of the fitting function. Furthermore, it is limited to a single varying parameter.

The design of antennas, used for applications such as feeds in reflector systems, requires the knowledge of the radiation pattern over a wide angular region in order to derive performance metrics such as aperture efficiency and crosspolarization. The efficiency (in terms of computational cost) with which such antennas are designed could be improved by means of parametric models of full radiation patterns. Furthermore, the design of an antenna usually involves a characterisation of radiation performance as well as reflection coefficients. The design cycle of a general antenna can be substantially improved if parametric models of the full pattern are combined with parametric macromodel representations of scattering ( $S$-parameters) or admittance responses [9][13], such that the antenna characteristics can be completely designed from fast analytical models.

This paper proposes an efficient parametric modeling technique to model the radiation pattern as well as reflection coefficients of a general antenna. The radiation pattern is modeled over a wide angular region, of up to a full sphere, as a function of the geometry of the antenna, taking into account more than one varying parameter. In the context of the MBPE paradigm [1], the proposed technique falls within the waveform-domain class, but with an added capability of providing a parametric description of the radiation pattern as a function of the antenna's geometry. Note that no attempt is made to interpolate over the angular variation of the pattern, as this part of the problem may be handled separately using 
the methods in, for instance, [1] and [4]. The proposed method combines characteristic basis function patterns (CBFPs) [14][16] with parameterization and interpolation steps to yield a parametric model that can be used to rapidly evaluate radiation characteristics for different combinations of geometric parameters of the antenna. The parameterization is achieved, differently from [8], by projecting the expansion coefficients (from estimation data) into the same subspace using a common and well-conditioned pivot matrix. The coefficients, obtained from this orthogonal projection, have a parameter dependence with smooth variation, and well defined trajectories, over a constrained multi-dimensional design space. Furthermore, in order to provide a full characterization of the antenna, a rational function representation, in pole-residue form, is utilized to model the antenna's $S$-parameters using the vector fitting (VF) method [17]. The poles and residues are then indirectly parameterized by first deriving a state-space realization of the rational function, followed by an appropriate similarity transform such that the variation of the state-space matrices is smooth over the design space [11]. The parametric models are generated from a limited set of EM simulations of the antenna under consideration. A combination of such parametric models may then be used to efficiently carry out typical antenna design activities requiring repeated EM simulations such as optimization, where the surrogate model may be used in a formal surrogate based optimization (SBO) procedure [18], [19], and sensitivity analysis.

This paper is organized as follows: Sec. II gives a brief description of the CBFP method, while Sec. III describes our main contributions, namely the parametric modeling algorithm for both radiation patterns and $S$-parameters of antennas. Sec. IV provides two examples illustrating the effectiveness of the presented models for different classes of antennas and finally, a conclusion is given in Sec. V.

\section{Characteristic Basis Function Patterns}

The CBFP method is described in [14] as a method to efficiently evaluate radiation patterns of antenna arrays. It is first applied to a single beam reflector antenna in [15]. Following the discussion in [14], [15], the CBFP method consists of reconstructing a radiation pattern as a linear combination of a few basis functions as

$$
\boldsymbol{F}_{r}(\Omega, s)=\sum_{n=1}^{N} \alpha_{n} \boldsymbol{f}_{n}(\Omega, s),
$$

where $\boldsymbol{F}_{r}(\Omega, s) \in \mathbb{C}^{N_{p} \times 1}$ is the approximated pattern in a general direction $\Omega$ and at a frequency $s$. The symbol $\boldsymbol{f}_{n}(\Omega, s) \in \mathbb{C}^{N_{p} \times 1}$ denotes the physics-based CBFPs, which may be given by an analytical pattern model, or are directly obtained from EM simulations, or measurements, and $N$ denotes the number of CBFPs. We will use the results of EM simulations as CBFPs throughout this paper. Note that $N_{p}=N_{\theta} N_{\phi}$, where $N_{\theta}$ and $N_{\phi}$ are the number of farfield points in the $\theta$ - and $\phi$-directions respectively, with $\theta$ denoting the polar angle and $\phi$ the azimuthal angle in a standard spherical coordinate system.
The objective of the CBFP method is thus to solve for the complex-valued coefficients $\alpha_{n}$ in (1) in order to determine an unknown pattern $\boldsymbol{F}_{r}(\Omega, s)$.

\section{A. Generating CBFPs}

The CBFPs are generated by EM simulations of a limited set of a combination of geometric parameters of the antenna. This set is herein referred to as the estimation set.

The objective here is to generate a minimum set of $N$ CBFPs that covers the design space of interest in the most efficient manner. The estimation set is generated either as a regular grid (for models with low dimension) or as a scattered grid by means of a design space filling rule or a latin hypercube sampling (LHS) [20] method augmented by the corner points of the design space.

\section{B. Solving Model Coefficients}

The CBFP method was developed mainly for rapid estimation of the pattern for calibration purposes [14], [15]. In that context, the coefficients $\alpha_{n}$, in (1), are obtained by taking $M$ measurements of $\boldsymbol{F}_{r}(\Omega, s)$ and solving the resulting system of linear equations either directly by using the Moore-Penrose pseudo-inverse or by orthogonalising the basis functions, by means of a singular value decomposition (SVD), as a preprocessing step to enhance modeling accuracy [21].

Let $\mathbf{F}_{\mathrm{M}}$ be an $N_{p} \times N$ matrix whose columns are the CBFPs in (1). In applying SVD, only the first $N_{R}\left(N_{R} \leqslant N\right)$ leftsingular vectors of $\mathbf{F}_{\mathrm{M}}$ are retained, constituting a (possibly) reduced set of CBFPs, denoted by $\mathbf{U}_{\mathrm{R}} \in \mathbb{C}^{N_{p} \times N_{R}}$. These vectors correspond to the singular values of $\mathbf{F}_{\mathrm{M}}$ above a certain threshold value, $\epsilon$, relative to the largest singular value [15]. The model coefficients, for each frequency $s$, are then given by

$$
\boldsymbol{\alpha}_{v}=\left[\mathbf{U}_{\mathrm{R}}\left(\Omega_{R}\right)\right]^{\dagger} \boldsymbol{F}_{r}\left(\Omega_{R}\right),
$$

where $\Omega_{R}$ are the $N_{R}$ distinct directions in which measurements of the yet to be determined pattern $\boldsymbol{F}_{r}(\Omega)$ are taken, and the superscript ${ }^{\dagger}$ indicates the pseudo-inverse of a matrix. The recovered full pattern is then given by

$$
\begin{aligned}
\boldsymbol{F}_{r}(\Omega) & =\mathbf{R}(\Omega) \boldsymbol{\alpha}_{v}, \\
\mathbf{R}(\Omega) & =\left[\boldsymbol{r}_{1}(\Omega), \boldsymbol{r}_{2}(\Omega), \cdots, \boldsymbol{r}_{N_{R}}(\Omega)\right],
\end{aligned}
$$

where $\mathbf{R}(\Omega) \in \mathbb{C}^{N_{p} \times N_{R}}$ is a matrix whose columns are given by

$$
\boldsymbol{r}_{k}(\Omega)=\frac{1}{\sigma_{k}} \mathbf{F}_{\mathrm{M}}(\Omega) \boldsymbol{V}_{k}(\Omega),
$$

where $\sigma_{k}$ is the $k^{t h}$ singular value of $\mathbf{F}_{\mathrm{M}}(\Omega)$ and $\boldsymbol{V}_{k}(\Omega) \in$ $\mathbb{C}^{N \times 1}$ is the corresponding $k^{\text {th }}$ column of the right singular matrix $\mathbf{V}(\Omega) \in \mathbb{C}^{N \times N_{R}}$, resulting from the SVD of $\mathbf{F}_{\mathrm{M}}(\Omega)$.

An advantage of solving for expansion coefficients using (2), is that $\boldsymbol{\alpha}_{v} \in \mathbb{C}^{N_{R} \times 1}$ is unique, with non-zero entries, for each $\boldsymbol{F}_{r}$. This is a critical property for the parametric modeling algorithm described in this paper. The primary aim of this paper is to predict values of $\boldsymbol{\alpha}_{v}$ as a function of the design space variables without a priori knowledge of $\boldsymbol{F}_{r}$, and this is addressed in what follows. Note that $N_{R}=N$ is used throughout the rest of this paper. 


\section{Parametric Modeling Algorithm}

The proposed method aims at constructing analytical models of the radiation patterns (over a wide angular region) as well as $S$-parameters of an antenna as a function of its variables such as geometrical dimensions or substrate features.

\section{A. Radiation Pattern modeling}

The problem at hand consists of accurately predicting radiation patterns corresponding to an arbitrary set of geometric parameters $\boldsymbol{x}^{*}$, within a given design space $\mathcal{D}$. The CBFP method is a model-based pattern prediction method and is efficient as it typically has a small number of solvable parameters.

Recently, a matrix projection based method [16] was proposed to obtain expansion weights at multiple frequencies without explicitly solving (1) at each frequency of interest. The methods in [8], [16] are limited to one dimensional parameter variations. To be more useful in a practical antenna design, the radiation pattern models [8] need to be extended to account for the variation of multiple geometric parameters. The main contribution herein is the extension to the general $\mathrm{N}$-dimensional parametric modeling case, and this is presented in what follows.

Consider a limited estimation set of Q points

$$
\mathcal{X}^{\text {est }}=\left\{\boldsymbol{x}_{1}, \boldsymbol{x}_{2}, \cdots, \boldsymbol{x}_{Q}\right\},
$$

and a corresponding set of CBFPs

$$
\mathcal{F}^{\text {est }}=\left\{\boldsymbol{f}_{1}^{e}(\Omega, s), \boldsymbol{f}_{2}^{e}(\Omega, s), \cdots, \boldsymbol{f}_{Q}^{e}(\Omega, s)\right\},
$$

where $\boldsymbol{x}_{k}$ denotes a vector of geometric parameters of the antenna, $\boldsymbol{f}_{k}^{e}(\Omega, s)$ is the corresponding radiation pattern and $\mathcal{X}^{\text {est }} \in \mathcal{D}$.

Starting from $Q$ basis functions in (6), a $Q \times Q$ matrix, $\mathbf{W}\left(s_{j}\right)$, of expansion coefficients is generated for each frequency $s_{j}$ by sampling each of the $Q$ CBFPs at $Q$ distinct points, $\Omega_{s}$, and solving (1) using the SVD method. That is

$$
\mathbf{W}\left(s_{j}\right)=\left[\boldsymbol{w}_{1}\left(s_{j}\right), \boldsymbol{w}_{2}\left(s_{j}\right), \cdots, \boldsymbol{w}_{Q}\left(s_{j}\right)\right],
$$

where $\boldsymbol{w}_{k}\left(s_{j}\right)$ is a column vector of expansion coefficients corresponding to the solution for the $k^{\text {th }}$ basis function $(k \in\{1,2, \cdots, Q\})$. The vector $\boldsymbol{w}_{k}\left(s_{j}\right)$, in (7), is obtained by replacing $\boldsymbol{F}_{r}(\Omega)$, in (2), by the actual basis function $\boldsymbol{f}_{k}^{e}(\Omega, s)$ at a frequency $s=s_{j}$ as

$$
\boldsymbol{w}_{k}\left(s_{j}\right)=\left[\mathbf{U}_{\mathrm{R}}\left(\Omega_{s}, s_{j}\right)\right]^{-1} \boldsymbol{f}_{k}^{e}\left(\Omega_{s}, s_{j}\right) .
$$

$\mathbf{U}_{R}$ is the left-singular matrix of the SVD realisation of a column stacked matrix of CBFPs as explained in Sec. II-B. We stress that it is important that the SVD be used to obtain each $\boldsymbol{w}_{k}\left(s_{j}\right)$, as a direct use of the Moore-Penrose pseudo-inverse would yield the trivial result that $\mathbf{W}\left(s_{j}\right)$, in (7), is an identity matrix of order $Q$. Utilising SVD results in each $\boldsymbol{w}_{k}\left(s_{j}\right)$ being a unique vector with no zero entry, effectively linking each unique point in the estimation set to a unique vector of expansion coefficients, which is necessary for parameterization.

Equation (8) can, in fact, be interpreted as an orthogonal projection of the radiation patterns in (6) into a subspace, $\mathcal{W}$, that is spanned by the columns of the matrix $\mathbf{W}$ in
(7). The orthogonal projection is carried out using a common matrix $\mathbf{U}_{\mathrm{R}}$ for all estimation points. This results in the CBFP expansion coefficients, in the subspace $\mathcal{W}$, having a smooth geometry-dependent variation over the constrained design space of interest, such that the expansion coefficients at an arbitrary point in the design space can be obtained by multivariate interpolation of the columns of (7) using, for example, simplicial methods. Therefore, (3a) is reformulated as

$$
\boldsymbol{F}_{r}(\Omega, s)=\mathbf{R}(\Omega, s) \boldsymbol{\alpha}_{v}\left(\boldsymbol{x}^{*}\right),
$$

where, for a one-dimensional parametric modeling problem,

$$
\boldsymbol{\alpha}_{v}\left(\boldsymbol{x}^{*}\right)=\sum_{k=1}^{Q} \boldsymbol{T}_{x_{k}} \ell_{k}\left(\boldsymbol{x}^{*}\right)
$$

and $\boldsymbol{x}^{*}$ is a point within the design space $\mathcal{D}$ where the radiation pattern is to be evaluated. The symbol $\boldsymbol{T}_{x_{k}}$ represents the known interpolation nodes given by the expansion coefficients computed in (7) and the interpolation kernel $\ell_{k}\left(\boldsymbol{x}^{*}\right)$ is a scalar function with properties

$$
\begin{aligned}
& \ell_{k}\left(\boldsymbol{x}_{i}\right)=1 \quad \text { if } \boldsymbol{x}_{i} \in \mathcal{X}^{\text {est }}, \\
& \ell_{k}\left(\boldsymbol{x}_{i}\right) \geq 0 .
\end{aligned}
$$

For instance, $\ell_{k}\left(\boldsymbol{x}^{*}\right)$ can be chosen as a piecewise linear function or a cubic spline whose robust implementations are readily available in MATLAB [22].

For the $N$-dimensional parametric modeling case, $\boldsymbol{\alpha}_{v}\left(\boldsymbol{x}^{*}\right)$ can be expressed as

$$
\boldsymbol{\alpha}_{v}\left(\boldsymbol{x}^{*}\right)=\sum_{k_{1}=1}^{Q} \cdots \sum_{k_{N}=1}^{Q} \boldsymbol{T}_{\left(x_{k_{1}} \cdots x_{k_{N}}\right)} \ell_{k_{1}}\left(x_{1}^{*}\right) \cdots \ell_{k_{N}}\left(x_{N}^{*}\right),
$$

where $\boldsymbol{x}^{*}=\left[x_{1}^{*}, \cdots, x_{N}^{*}\right]$ and the kernel functions $\ell_{k_{i}}(\cdot)$ satisfy (11).

The result in (10) is similar to the technique in [8] with the difference being that the accuracy of the calculated expansion coefficients depends on $\mathbf{U}_{R}$ being well conditioned rather than on a judicious choice of a fitting function (see Fig. 4 in Sec. IV-A). The multivariate representation, in (12), is our main contribution and extends the one dimensional method in [8].

The pattern modeling can be accomplished by having two sets of CBFPs: one consisting of the $\theta$-component of the radiated electric far-field $\mathcal{F}_{\theta}^{\text {est }}$, and the other set consists of the $\phi$-component of the radiated far-field $\mathcal{F}_{\phi}^{\text {est }}$. The modeling steps (7)-(12) are then performed separately using $\mathcal{F}_{\theta}^{\text {est }}$ and $\mathcal{F}_{\phi}^{\text {est }}$ respectively and the resulting full radiated far-field may then be obtained.

\section{B. S-Parameter modeling}

A set of $S$-parameters is obtained from EM simulations for each point in the estimation set in (5). Since the number of points in the estimation set is typically small, statistical modeling techniques such as Kriging, co-Kriging or neural networks [18], [19], [23] are not suitable to accurately model the $S$-parameter variations over the design space of interest. Instead, parametric macromodeling methods are used in this section. 
The key concepts in parametric macromodeling techniques are repeated here for completeness, with more in depth discussions available in [9]-[13] and the references therein. We will limit the discussion of $S$-parameter modeling to reflection coefficients. However, the macromodeling technique can be readily applied to model an entire $P \times P$ scattering matrix, with $P$ being the number of electrical ports.

Consider a set of $Q S$-parameter vectors

$$
\mathcal{H}=\left\{\boldsymbol{h}_{\boldsymbol{x}_{1}}(s), \boldsymbol{h}_{\boldsymbol{x}_{2}}(s), \cdots, \boldsymbol{h}_{\boldsymbol{x}_{Q}}(s)\right\},
$$

with each $\boldsymbol{h}_{\boldsymbol{x}_{i}}(s)$ being a function of frequency and corresponding to the antenna's reflection coefficients for an input vector $\boldsymbol{x}_{i} \in \mathcal{X}^{e s t}$.

The first step in the algorithm consists of fitting each $\boldsymbol{h}_{\boldsymbol{x}_{i}}(s)$ with a frequency-dependent rational transfer function of the form

$$
H_{\boldsymbol{x}_{i}}^{p}(s)=r_{0}+\sum_{n=1}^{K} \frac{r_{n}}{s-z_{n}},
$$

where $r_{0}$ is the direct coupling constant, $r_{n}$ are the residues and $z_{n}$ are the system's poles, which are determined iteratively using vector fitting [17], and $K$ is the order of the approximation. Note that $r_{0}, r_{n}$ and $z_{n}$ are dependent on the design space point $\boldsymbol{x}_{i}$ in the estimation set (5). The expression in (14) is then converted to a Gilbert state-space realisation [24], where the transfer function is given by

$$
H_{\boldsymbol{x}_{i}}(s)=\mathbf{C}_{\boldsymbol{x}_{i}}\left(s \mathbf{I}-\mathbf{A}_{\boldsymbol{x}_{i}}\right)^{-1} \mathbf{B}_{\boldsymbol{x}_{i}}+\mathbf{D}_{\boldsymbol{x}_{i}},
$$

and the matrices $\mathbf{A}_{\boldsymbol{x}_{i}} \in \mathbb{C}^{K \times K}$ is a diagonal matrix of poles, $\mathbf{C}_{\boldsymbol{x}_{i}} \in \mathbb{C}^{1 \times K}$ is a row vector of residues, and $\mathbf{B}_{\boldsymbol{x}_{i}} \in \mathbb{N}^{K \times 1}$ is a unity vector while $\mathbf{D}_{\boldsymbol{x}_{i}} \in \mathbb{R}$ equals the direct coupling term $r_{0}$. The expression in (15) is henceforth referred to as a root macromodel, and all root macromodels $H_{\boldsymbol{x}_{i}}(s)$, with $\boldsymbol{x}_{i} \in \mathcal{X}^{\text {est }}$, have the same order $K$. The parametric macromodel may then be obtained by interpolation of the state-space matrices as

$$
\widetilde{H}\left(\boldsymbol{x}^{*}, s\right)=\sum_{k=1}^{Q} \boldsymbol{T}_{x_{k}} \ell_{k}\left(\boldsymbol{x}^{*}\right),
$$

where $\boldsymbol{T}_{x_{k}}$ represents a state-space matrix $\left(\mathbf{A}_{\boldsymbol{x}_{k}}, \mathbf{B}_{\boldsymbol{x}_{k}}, \mathbf{C}_{\boldsymbol{x}_{k}}\right.$ or $\mathbf{D}_{\boldsymbol{x}_{k}}$ ) and $\ell_{k}\left(\boldsymbol{x}^{*}\right)$ is the interpolation kernel satisfying (11).

However, the interpolation of Gilbert state-space matrices may suffer from a non-smooth variation of the state-space matrices in the design space $\mathcal{D}$, and this may lead to inaccurate results [11]. A more accurate parametric macromodel is obtained by converting the pole-residue transfer function in (14) into the barycentric form [13] as

$$
H_{\boldsymbol{x}_{i}}^{b}(s)=\frac{\boldsymbol{F}_{0}+\sum_{n=1}^{K} \boldsymbol{F}_{n} \phi_{n}(s)}{f_{0}+\sum_{n=1}^{K} f_{n} \phi_{n}(s)},
$$

where the frequency-dependent basis function is defined as

$$
\phi_{n}(s)=\frac{1}{s-a_{n}}
$$

and the system's poles, $a_{n}$, are fixed and independent of any design space point $\boldsymbol{x}_{i}$.
The parameters of the numerator and denominator of (17) are found by imposing that $H_{\boldsymbol{x}_{i}}^{b}(s)=H_{\boldsymbol{x}_{i}}^{p}(s)$, which yields the following conditions:

$$
\begin{gathered}
\sum_{n=1}^{K} f_{n} \prod_{\substack{n^{\prime}=1 \\
n^{\prime} \neq n}}^{K}\left(z_{l}-a_{n^{\prime}}\right)=-f_{0} \prod_{n^{\prime}=1}^{K}\left(z_{l}-a_{n^{\prime}}\right) \quad \text { for } l=1, \cdots, K \\
\boldsymbol{F}_{n}=f_{n} \cdot H_{\boldsymbol{x}_{i}}^{p}\left(a_{n}\right) \quad \text { for } n=1, \cdots, K \\
\boldsymbol{F}_{0}=f_{0} \cdot r_{0}
\end{gathered}
$$

The proof for (19) can be found in [13].

By fixing the value of $f_{0}$ in (19), values of $f_{n}$ can be found by solving the resulting system of linear equations. Condition (20) originates from setting $H_{\boldsymbol{x}_{i}}^{b}\left(a_{n}\right)=H_{\boldsymbol{x}_{i}}^{p}\left(a_{n}\right)$ and the coefficients $\boldsymbol{F}_{n}$ can thus be solved. The final condition is found by imposing the equality of the pole-residue and barycentric transfer functions as $s \rightarrow \infty$.

Having computed the coefficients of the transfer function in (17), a state-space realization can then be obtained by first deriving state-space matrices $\left\{\mathbf{A}_{\boldsymbol{x}_{i}}^{\text {num }}, \mathbf{B}_{\boldsymbol{x}_{i}}^{\text {num }}, \mathbf{C}_{\boldsymbol{x}_{i}}^{\text {num }}, \mathbf{D}_{\boldsymbol{x}_{i}}^{\text {num }}\right\}$ and $\left\{\mathbf{A}_{\boldsymbol{x}_{i}}^{\text {den }}, \mathbf{B}_{\boldsymbol{x}_{i}}^{\text {den }}, \mathbf{C}_{\boldsymbol{x}_{i}}^{\text {den }}, \mathbf{D}_{\boldsymbol{x}_{i}}^{\text {den }}\right\}$ for the numerator and denominator of (17) respectively [11], [13]. The state matrices are given by

$$
\begin{aligned}
\mathbf{A}_{\boldsymbol{x}_{i}}^{\text {num }} & =\mathbf{A}_{\boldsymbol{x}_{i}}^{\text {den }}=\operatorname{blkdiag}\left\{a_{n} \mathbf{I}_{P}\right\}, \\
\mathbf{B}_{\boldsymbol{x}_{i}}^{\text {num }} & =\mathbf{B}_{\boldsymbol{x}_{i}}^{\text {den }}=\left[\mathbf{I}_{P}, \cdots, \mathbf{I}_{P}\right]^{T}, \\
\mathbf{C}_{\boldsymbol{x}_{i}}^{\text {num }} & =\left[\boldsymbol{F}_{1}, \cdots, \boldsymbol{F}_{K}\right], \\
\mathbf{C}_{\boldsymbol{x}_{i}}^{\text {den }} & =\left[f_{1} \mathbf{I}_{P}, \cdots, f_{K} \mathbf{I}_{P}\right], \\
\mathbf{D}_{\boldsymbol{x}_{i}}^{\text {num }} & =\boldsymbol{F}_{0}, \\
\mathbf{D}_{\boldsymbol{x}_{i}}^{\text {den }} & =f_{0} \mathbf{I}_{P},
\end{aligned}
$$

where $n=1, \cdots, K, \mathbf{I}_{P}$ is an identity matrix of order $P$ and the superscript ${ }^{T}$ denotes the transpose operator. The final state-space matrices of the barycentric transfer function (17) are then obtained, after some algebraic manipulations, as

$$
\begin{aligned}
\widetilde{\mathbf{A}}_{\boldsymbol{x}_{i}} & =\mathbf{A}_{\boldsymbol{x}_{i}}^{\text {den }}-\mathbf{B}_{\boldsymbol{x}_{i}}^{\text {den }}\left(\mathbf{D}_{\boldsymbol{x}_{i}}^{d e n}\right)^{-1} \mathbf{C}_{\boldsymbol{x}_{i}}^{d e n}, \\
\widetilde{\mathbf{B}}_{\boldsymbol{x}_{i}} & =\mathbf{B}_{\boldsymbol{x}_{i}}^{\text {den }}\left(\mathbf{D}_{\boldsymbol{x}_{i}}^{\text {den }}\right)^{-1}, \\
\widetilde{\mathbf{C}}_{\boldsymbol{x}_{i}} & =\mathbf{C}_{\boldsymbol{x}_{i}}^{\text {num }}-\mathbf{D}_{\boldsymbol{x}_{i}}^{\text {num }}\left(\mathbf{D}_{\boldsymbol{x}_{i}}^{d e n}\right)^{-1} \mathbf{C}_{\boldsymbol{x}_{i}}^{\text {den }}, \\
\widetilde{\mathbf{D}}_{\boldsymbol{x}_{i}} & =\mathbf{D}_{\boldsymbol{x}_{i}}^{\text {num }}\left(\mathbf{D}_{\boldsymbol{x}_{i}}^{\text {den }}\right)^{-1} .
\end{aligned}
$$

An accurate parametric model, within the design space $\mathcal{D}$, may then be obtained by interpolating the state-space matrices (23a)-(23d) by using (16).

\section{Method Complexity}

The number of EM simulations required in the modeling process depends on the number of parameters involved and on the dynamic behaviour over the design space. However, the overall number is typically much smaller when compared to statistical methods.

The pattern modeling algorithm (Sec. III-A) involves simple algebraic operations such as SVD and matrix inversions (of very small matrices), and a serial (as opposed to parallel) implementation provides sufficiently fast model building and 
evaluation times. E.g., a model with 49 CBFPs (over a full sphere with $N_{\theta}=181$ and $N_{\phi}=361$ ) is built in 11.89 seconds per frequency point, and evaluated in 0.32 seconds (see Sec. IV for details on the computing platform). However, the building time can be significantly reduced as the algorithm lends itself to a certain degree of parallelism:

1) The computation of each column, $\boldsymbol{w}_{k}\left(s_{j}\right)$, of $\mathbf{W}\left(s_{j}\right)$ in (7) is independent from all other columns and can therefore be done in parallel.

2) The matrix $\mathbf{W}\left(s_{j}\right)$ can be computed in parallel for each frequency $s_{j}$.

The $S$-parameter modeling algorithm in Sec. III-B is typically an order of magnitude faster compared to the pattern modeling algorithm, both in terms of model construction and evaluation. Furthermore, it also lends itself to some level of parallelism as the construction of root macromodels is done independently for each point in the design space.

Both algorithms in Sec. III can be executed on estimation points in a uniform or scattered grid.

\section{NuMERICAL EXAMPLES}

In this section, the method proposed in Sec. III is validated through some examples with different antennas, illustrating the versatility of the method. The worst case root mean square error $\left(\varepsilon_{\max }^{\mathrm{RMS}}\right)$, between the parametric models and the validation data samples, is chosen to assess the quality of both the pattern and return loss models. The error metric is given by

$$
\begin{aligned}
\varepsilon^{\mathrm{RMS}}(\boldsymbol{x}) & =\sqrt{\frac{\sum_{k=1}^{N_{d}}\left|G_{k}(\boldsymbol{x})-\widetilde{G}_{k}(\boldsymbol{x})\right|^{2}}{N_{d}}}, \\
\varepsilon_{\max }^{\mathrm{RMS}} & =\varepsilon^{\mathrm{RMS}}\left(\boldsymbol{x}_{m}\right),
\end{aligned}
$$

where $\boldsymbol{x}$ is a point in the validation set and $G_{k}(\boldsymbol{x})$ is the validation data, $\widetilde{G}_{k}(\boldsymbol{x})$ is the prediction from the model, $N_{d}$ is the number of sample points and $\boldsymbol{x}_{m}$ is the point in the validation set that maximises (24).

All experiments are performed using MATLAB [22], on a 64-bit Windows platform with an Intel Core i7 - $2600 \mathrm{CPU}$ at $3.4 \mathrm{GHz}$ and $16 \mathrm{~GB}$ of RAM.

\section{A. Dipole Antenna}

The effect of varying the length of a vertical dipole antenna, radiating in free space, is modeled in this example. This example is similar to the modeling example considered in [5], where we model the effects of varying the length of the dipole on the pattern at one frequency instead of modeling the frequency dependence of the pattern of a fixed length dipole as was done in [5]. Our method, however, does not attempt to interpolate the spatial distribution of the radiation pattern, as this is normally fast to calculate once the current distribution on the antenna is known.

The length of the antenna is given as $l=x \lambda_{0}$, where $\lambda_{0}$ is the wavelength at $f_{0}=842.86 \mathrm{MHz}$, and $x \in[0.5,1.5]$. The estimation set consists of 15 points uniformly sampled in the range $[0.5,1.5]$. The model is validated at 20 randomly generated points (not in the estimation set) within the modeling range. All EM simulations are done using FEKO [25], a commercial Method of Moments (MoM) solver.

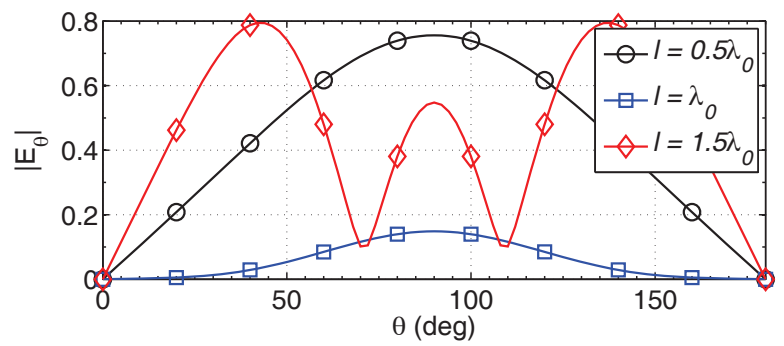

Fig. 1. Selected basis functions of the dipole from the $\mathcal{F}_{\theta}^{\text {est }}$ in the $\phi=90^{\circ}$ plane. Only 3 CBFPs are shown for clarity and to illustrate pattern variation for different dipole lengths.

The CBFPs in this example are $\mathcal{F}_{\theta}^{e s t}$ and $\mathcal{F}_{\phi}^{e s t}$ for each point in the estimation set, as explained in Sec. III-A, and a plot of the CBFPs is shown in Fig. 1, revealing a non-smooth variation of the radiation pattern as a function of $l$ and thus making a direct interpolation prone to inaccuracies. However, a smooth variation of the expansion coefficients, as shown in Fig. 2, allows an accurate recovery of the full pattern using (9).

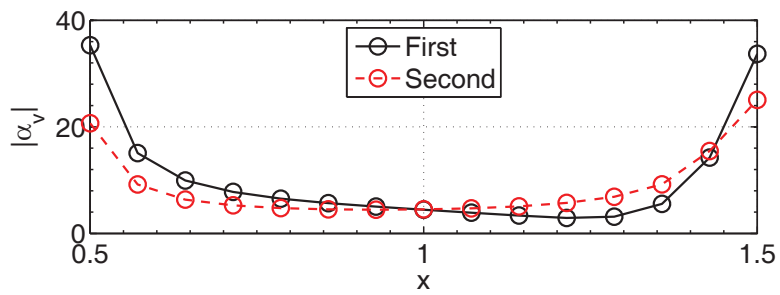

Fig. 2. Variation of the magnitude of the first two entries of the expansion coefficients as a function of the estimation points. Only two entries are shown for clarity and also because they dominate the remaining entries in terms of magnitude. The markers (o) denote the estimation points.
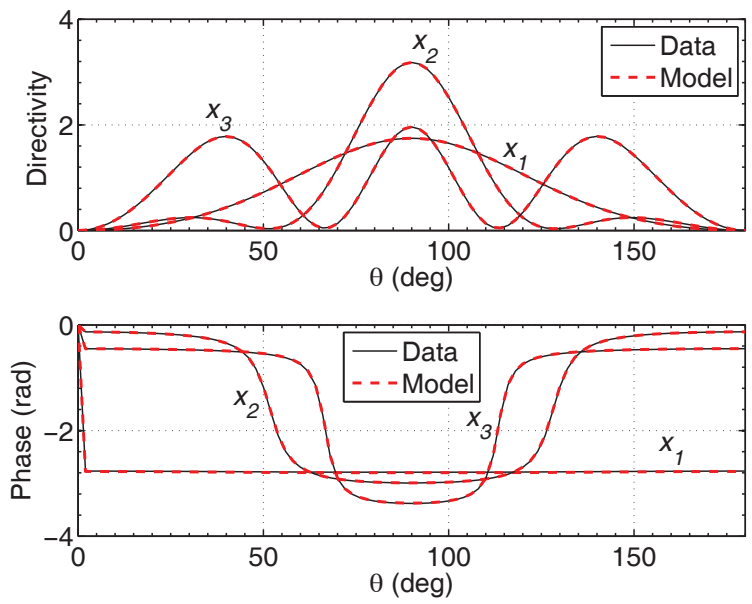

Fig. 3. Directivity variation (top panel) and phase variation (bottom panel) of the corresponding radiated co-polarised electric field, in the $\phi=90^{\circ}$ plane, for different points in the validation set, with dipole lengths $x_{1}=$ $0.625 \lambda_{0}, x_{2}=1.225 \lambda_{0}$ and $x_{3}=1.425 \lambda_{0}$. The patterns are modeled at $f_{0}=842.86 \mathrm{MHz}$. 
A comparison between the modeled and actual pattern is shown in Fig. 3 for a few points in the validation set, where the increase in directivity and side lobe levels can be clearly seen as the length of the dipole increases. A cubic spline is used as the interpolation kernel. The maximum magnitude and phase modeling errors in Fig. 3 are $\varepsilon^{M}=2.6 \times 10^{-3} \mathrm{~V} / \mathrm{m}$ and $\varepsilon^{\Phi}=0.011$ radians respectively. Note that the proposed model has only 15 solvable parameters compared to 56 parameters required for the example in [5], thus demonstrating the compactness of the proposed pattern model. A comparison with the technique in [8], with Legendre functions and high order polynomials being used as the parameter-dependent fitting functions, yields similar results to our proposed method at low frequencies $(<900 \mathrm{MHz})$. The results obtained with the method in [8] are less accurate at higher frequencies as shown in Fig. 4. Indeed, Fig. 4 demonstrates the robustness of our method, with respect to [8], when there is a large dynamic variation in the angular-dependent basis functions (CBFPs). Moreover, with the approach in [8] it can be observed, in Fig. 4 , that more accurate results are obtained when using a high order polynomial as a fitting function compared to the case where Legendre fitting functions are used. This confirms the strong dependence, of the method in [8], on the choice of the fitting function. It is difficult to select the best fitting function beforehand.

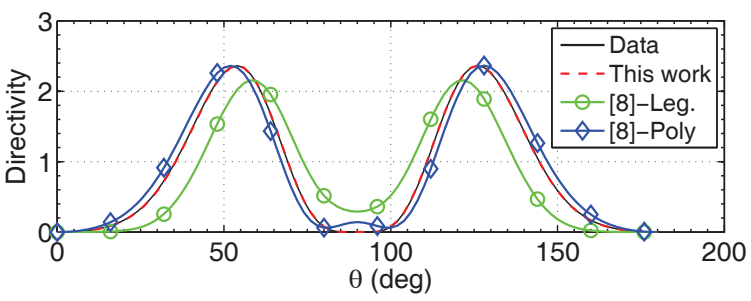

Fig. 4. Comparison of the modeling capabilities of the proposed approach and the method in [8], with two different choices of parameter-dependent functions namely: Legendre (Leg.) and polynomial (Poly) functions. The patterns are shown in the $\phi=90^{\circ}$ plane, at a frequency of $1.06 \mathrm{GHz}$.

The modeling of reflection coefficients is achieved with root macromodels (see Sec. III-B) of order 5, and the interpolation kernel is also a cubic spline function. Highly accurate modeling results are shown in Fig. 5, where the effects of increasing the length of the dipole can be clearly seen.

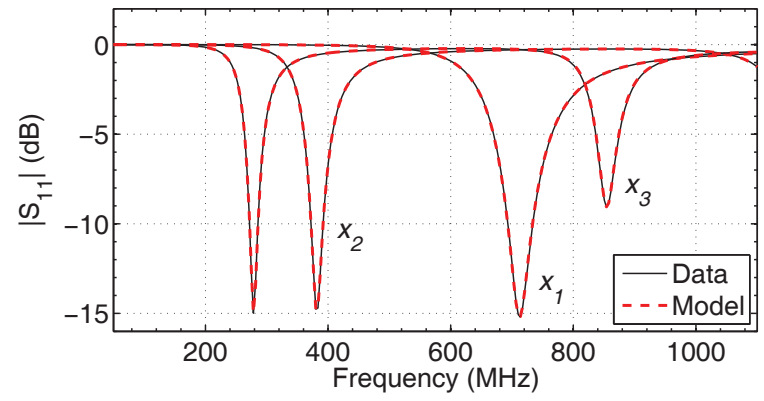

Fig. 5. Reflection coefficients at selected points in the validation set: $x_{1}=$ $0.575 \lambda_{0}, x_{2}=1.075 \lambda_{0}$ and $x_{3}=1.475 \lambda_{0}$. The appearance of a second resonant frequency within the simulated bandwidth can be seen as the length of the dipole increases beyond a wavelength.
The modeling error (25) for the entire radiation pattern, evaluated for frequencies in the range $[50,1100] \mathrm{MHz}$, is 0.1109 while the error for reflection coefficients is $9.4 \times 10^{-3}$.

\section{B. Axially Corrugated Choke Horn Antenna}

An axially corrugated horn antenna with three chokes is modeled in this example. The antenna is depicted in Fig. 6 and the design variables are listed in Table I, where $a_{c}$ is the complement of the horn's flare angle and $d_{c}$ is the diameter of the input waveguide. The width and depth of the corrugations are fixed at $0.120 \lambda_{0}$ and $0.273 \lambda_{0}$ respectively, where $\lambda_{0}$ is the wavelength at $f_{0}=1.5 \mathrm{GHz}$. The thickness of each ridge is fixed at $0.024 \lambda_{0}$.

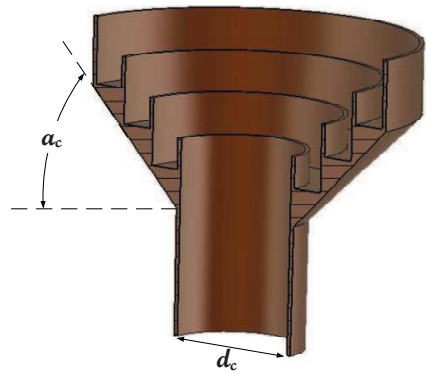

Fig. 6. Simulation model of an axially corrugated choke horn.

TABLE I

Design Parameters of the ChoKe Horn Antenna.

\begin{tabular}{ccc}
\hline Parameter & Min. & Max. \\
\hline$a_{c}$ & $0^{\circ}$ & $50^{\circ}$ \\
$d_{c}$ & $0.9 \lambda_{0}$ & $\lambda_{0}$ \\
Frequency & $0.867 f_{0}$ & $1.167 f_{0}$ \\
\hline
\end{tabular}

The estimation grid consists of a $6 \times 6$ grid of uniformly sampled points $\left(a_{c}, d_{c}\right)$ in the range specified in Table $\mathrm{I}$ and the validation samples consist of a $5 \times 5$ grid of points defined such that each validation point lies at the centre of a rectangle described by 4 of its nearest estimation points. The horn is simulated with the time domain solver in CST Microwave Studio (CST-MWS) [26] and the variation of CBFPs is shown in Fig. 7.

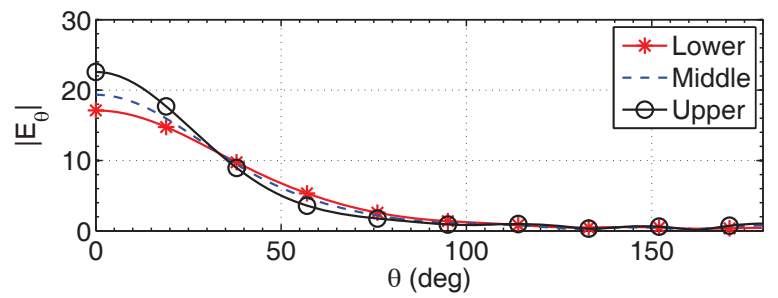

Fig. 7. Selected basis functions from the $\mathcal{F}_{\theta}^{e s t}$ set in the $\phi=90^{\circ}$ plane. Only 3 basis functions (at the lower $\left(0^{\circ}, 0.9 \lambda_{0}\right)$ and upper $\left(50^{\circ}, \lambda_{0}\right)$ corners as well as the middle $\left(20^{\circ}, 0.96 \lambda_{0}\right)$ of the design space) are shown for clarity.

The parametric model is constructed as detailed in Sec. III-A and a piecewise linear function is used as the interpolation kernel. The model is the evaluated at points in the 


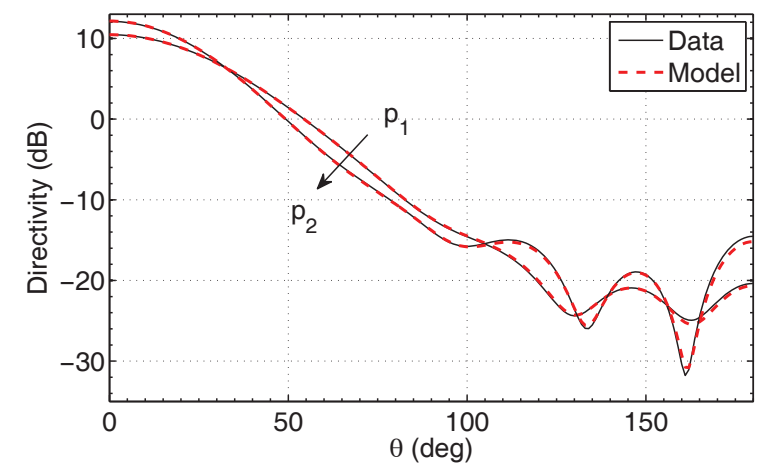

(a)

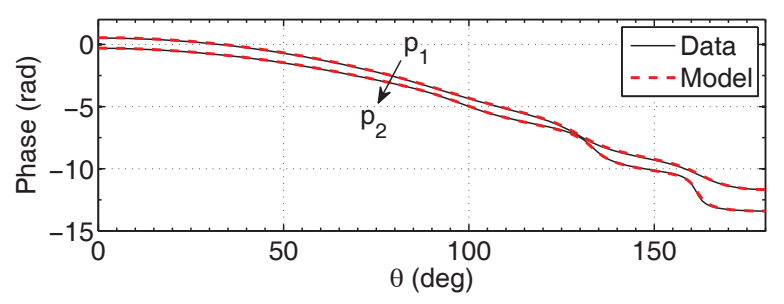

(b)

Fig. 8. (a) Directivity variation, (b) Phase variation across the design space for a fixed $d_{c}=19.8 \mathrm{~cm}$ while $a_{c}$ varies from $p_{1}$ to $p_{2}$ as $a_{c}=5^{\circ}$ and $a_{c}=45^{\circ}$ respectively. The patterns are evaluated at $1.3 \mathrm{GHz}$, in the $\phi=90^{\circ}$ plane and the increase in directivity is clear as the flare angle decreases.

validation sets and selected results are shown in Fig. 8 (with $\varepsilon^{M}=0.1049$ and $\varepsilon^{\Phi}=0.0347$ ) where the effect of increasing the flare angle's complement, $a_{c}$, can clearly be observed.

The maximum modeling error at the validation points is 0.29 and 0.1276 for magnitude and phase respectively. The variation of the modeling error as function of the design variables is shown in Fig. 9. The error plots reveal more dynamic variations of the radiation pattern with respect to the flare angle. However, the trajectories of the CBFP expansion coefficients are continuous across the design space such that the resulting model is accurate over the whole design space. This example validates the proposed pattern modeling algorithm even in the case of a large non-linear variation of the radiation patterns within the design space.

Modeling of the corresponding reflection coefficients requires a local approach as the frequency behavior is different, in terms of the presence of resonances and the general trend, in different regions of the design space (i.e., the trajectories of the state-space matrices are not smooth across such a large design space). Therefore, different fitting orders are used in different regions of the design space, with the number of poles varying from 5 to 8 , in order to yield relatively accurate results. A more dynamic variation of reflection coefficients as a function of the flare angle is observed in this example, and therefore the design space is divided in regions according to flare angle values, and each region is fitted with the appropriate number of poles. The variation of the modeling error in the design space is shown in Fig. 10 and a plot of the model with maximum error is shown in Fig. 11.

Errors, though very small, between validation data and models of reflection coefficients, in Fig. 11, could be reduced by using adaptive sampling methods such as the tree-based

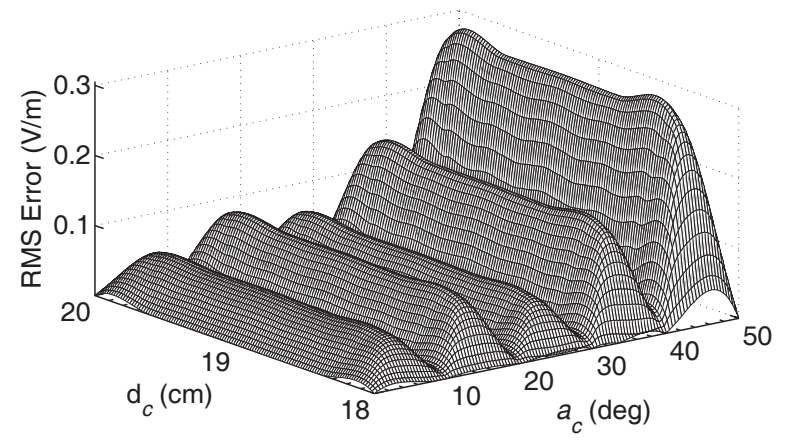

(a)

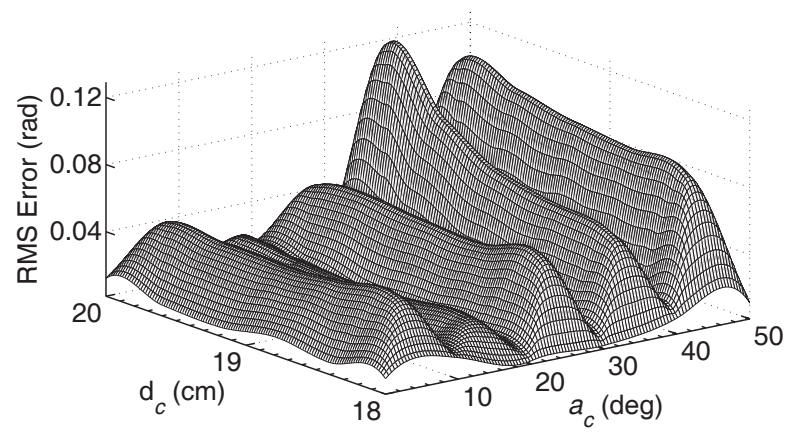

(b)

Fig. 9. Radiation pattern modeling: (a) Magnitude modeling error, (b) phase modeling error over the design space, at $1.75 \mathrm{GHz}$, where maximum errors occur.

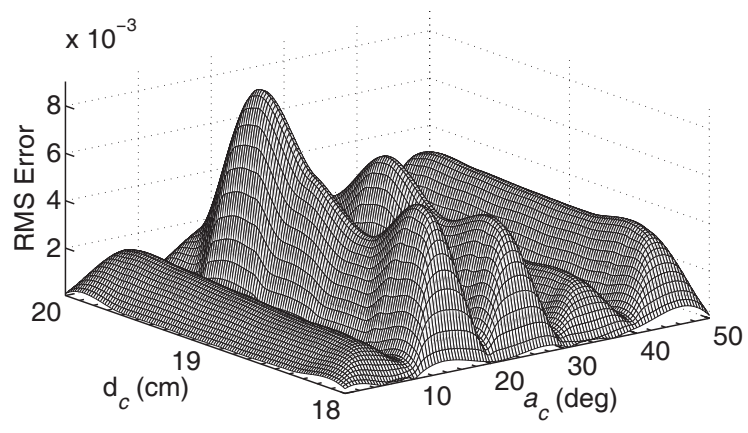

Fig. 10. S-parameter modeling error over the design space.

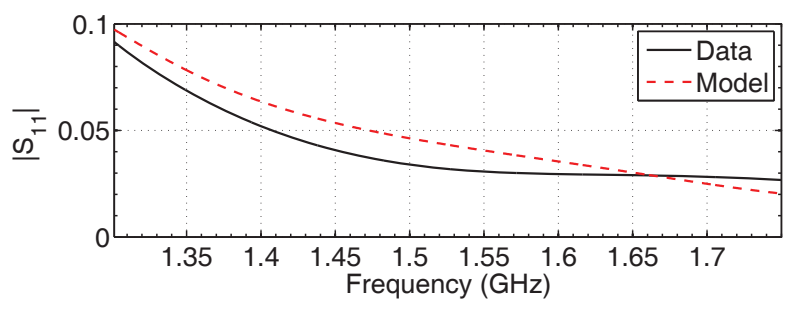

Fig. 11. Comparison between data and model at the point in the validation set with maximum error.

sequential technique in [27].

\section{Convergence Study of the CBFP Models}

In this section, we present some results concerning the accuracy of the CBFP models as a function of the number of points in the estimation set. To this end, we consider the example in Sec. IV-B and both regular and scattered grids are 
considered. For the scattered grid case, estimation points are selected upfront and adaptively according to a design space filling rule such that the design space coverage is maximised.

Models constructed with different numbers of estimation points are evaluated on a dense $9 \times 9$ grid of validation points and the results are presented in Table II, where a spline and linear interpolation kernels are used for regular and scattered grids respectively.

TABLE II

Pattern Modeling Mean RMS ERrors Over All Validation POINTS AS THE Number of ESTIMATION POINTS INCREASES FOR BOTH REGULAR AND SCATTERED GRIDS.

\begin{tabular}{cccccccc}
\hline \multicolumn{3}{c}{ Scattered Grid } & & \multicolumn{3}{c}{ Regular Grid } \\
\cline { 1 - 3 } \cline { 5 - 7 } Points & Magnitude & Phase & & Points & Magnitude & Phase \\
\cline { 1 - 4 } 13 & 0.0553 & 0.1189 & & 16 & 0.1214 & 0.1389 \\
25 & 0.0360 & 0.0950 & & 25 & 0.0972 & 0.0999 \\
34 & 0.0271 & 0.0766 & & 36 & 0.0101 & 0.0265 \\
48 & 0.0244 & 0.0691 & & 49 & 0.0441 & 0.0571 \\
\hline
\end{tabular}

The results in Table II generally reveal lower modeling errors for scattered grids compared to regular grids with a similar number of points. Furthermore, a monotonic decline in modeling errors is observed as the number of estimation points increases, with steady-state values occurring at around 36 estimation points. The slightly higher error for the case of 49 estimation points, in a regular grid, is due to Runge effects at the edge of the design space.

Table II generally shows a higher modeling error of phase compared to magnitude. This is because a larger number of estimation points are required to capture rapid phase variations for accuracy levels similar to the magnitude errors in Table II. This is illustrated in Fig. 2 where it may be inferred that fewer estimation points can be used due to the almost constant values of the magnitudes of expansion coefficients between $x \approx 0.7$ and $x \approx 1.3$. However, all 15 points were required to accurately capture the phase variations shown in Fig. 3 . Thus, for applications where only magnitudes of the radiated far-field are of interest, accurate models can be achieved with a smaller estimation set.

For cases where a rapid variation of the radiation pattern occurs across the design space, such as a large difference in directivity, a few extra estimation points may have to be allocated in the region of rapid variation, in order to obtain accurate results. A large difference in directivity may be caused by, for instance, a resonance in the radiating structure or feed of the antenna.

\section{CONClusion}

This paper presented a novel algorithm to efficiently model the full radiation pattern as well as reflection coefficients of an antenna as a function of its geometry. The proposed method models radiation patterns as a linear combination of CBFPs, where the expansion coefficients are parameterized with respect to multiple design variables. Reflection coefficients are modeled as rational functions where the poles and residues are indirectly parameterized. Accurate models are constructed from a limited set of EM simulations and can be used to efficiently carry out antenna design activities where multiple simulations are required. Numerical simulations confirm the accuracy of the proposed method for different antennas.

\section{REFERENCES}

[1] E. K. Miller, "Model-based parameter estimation in electromagnetics. I. Background and theoretical development," IEEE Antennas Propag. Mag., vol. 40, no. 1, pp. 42-52, Feb 1998.

[2] E. K. Miller, "Model-based parameter estimation in electromagnetics. II. Applications to EM observables," IEEE Antennas Propag. Mag., vol. 40, no. 2, pp. 51-65, Apr 1998.

[3] E. K. Miller, "Using adaptive estimation to minimize the number of samples needed to develop a radiation or scattering pattern to a specified uncertainty," ACES J., vol. 17, no. 3, pp. 176-186, Nov 2002.

[4] E. K. Miller, "Adaptive sparse sampling to estimate radiation and scattering patterns to a specified uncertainty with model-based parameter estimation: compute patterns using as few as two to four samples per lobe," IEEE Antennas Propag. Mag., vol. 57, no. 4, pp. 103-113, Aug 2015.

[5] D. H. Werner and R. J. Allard, "The simultaneous interpolation of antenna radiation patterns in both the spatial and frequency domains using modelbased parameter estimation," IEEE Trans. Antennas Propag., vol. 48, no. 3, pp. 383-392, Mar 2000.

[6] R. J. Allard and D. H. Werner, "The model-based parameter estimation of antenna radiation patterns using windowed interpolation and spherical harmonics," IEEE Trans. Antennas Propag., vol. 51, no. 8, pp. 18911906, Aug 2003.

[7] W. Dullaert and H. Rogier, "Novel compact model for the radiation pattern of UWB antennas using vector spherical and Slepian decomposition," IEEE Trans. Antennas Propag., vol. 58, no. 2, pp. 287-299, Feb 2010.

[8] M. A. Francavilla, G. Giordanengo, M. Righero, G. Vecchi, F. Vipiana, "Physics-based parametric interpolation," in 9th Eur. Conf. Antennas Propag. (EuCAP), Lisbon, Portugal, Apr. 2015, pp. 1-5.

[9] F. Ferranti, T. Dhaene, L. Knockaert, G. Antonini and A. Ciccomancini Scogna, "Scalable compact models for fast design optimization of complex electromagnetic systems," Int. J. RF Microw. Comput.-Aided Eng., SPECIAL ISSUE on EM-CAD Models and their Integration into IndustryStandard CAD Tools, vol. 221 no. 1, pp. 20-29, Jan 2012.

[10] F. Ferranti, L. Knockaert and T. Dhaene, "Passivity-Preserving Parametric Macromodeling by Means of Scaled and Shifted State-Space Systems," IEEE Trans. Microw. Theory Tech., vol. 59, no. 10, pp. 2394-2403, Oct 2011.

[11] E. R. Samuel, L. Knockaert, F. Ferranti and T. Dhaene, "Guaranteed passive parameterized macromodeling by using sylvester state-space realizations," IEEE Trans. Microw. Theory Tech., vol. 61, no. 4, pp. 14441454, Apr 2013.

[12] D. Deschrijver, T. Dhaene and D. De Zutter, "Robust Parametric Macromodeling Using Multivariate Orthonormal Vector Fitting," IEEE Trans. Microw. Theory Tech., vol. 56, no. 7, pp. 1661-1667, July 2008.

[13] P. Triverio, S. Grivet-Talocia and M. S. Nakhla, "A parameterized macromodeling strategy with uniform stability test," IEEE Trans. Adv. Packag., vol. 32, no. 1, pp. 205-215, Feb 2009.

[14] R. Maaskant, M. V. Ivashina, S. J. Wijnholds and K. F. Warnick, "Efficient prediction of array element patterns using physics-based expansions and a single far-field Measurement," IEEE Trans. Antennas Propag., vol. 60, no. 8, pp. 3614-3621, Aug 2012.

[15] A. Young, R. Maaskant, M. V. Ivashina, D. I. L. de Villiers and D. B. Davidson, "Accurate beam prediction through characteristic basis function patterns for the MeerKAT/SKA radio telescope antenna," IEEE Trans. Antennas Propag., vol. 61, no. 5, pp. 2466-2473, May 2013.

[16] N. Mutonkole and D. I. L. de Villiers, "characteristic basis function patterns method for reflector antenna calibration: an extension to multiple frequencies," in 9th Eur. Conf. Antennas Propag. (EuCAP), Lisbon, Portugal, Apr. 2015, pp. 1-5.

[17] B. Gustavsen and A. Semlyen, "Rational approximation of frequency domain responses by vector fitting," IEEE Trans. Power Del., vol. 14, no. 3, pp. 1052-1061, Jul 1999.

[18] I. Couckuyt, F. Declercq, T. Dhaene, H. Rogier and L. Knockaert, "Surrogate-based infill optimization applied to electromagnetic problems," Int. J. RF Microw. Comput.-Aided Eng., vol. 20, no. 5, pp. 492-501. [Online]. Available: http://dx.doi.org/10.1002/mmce.20455

[19] D. Gorissen, "Grid-enabled Adaptive Surrogate Modeling for Computer Aided Engineering," Ph.D. dissertation, Ghent University, Ghent, Belgium, 2010. 
[20] M. D. McKay, R. J. Beckman and W. J. Conover, "A Comparison of three methods for selecting values of input variables in the analysis of output from a computer code," Technometrics, vol. 42, no. 1, pp. 55-61, Feb 2000.

[21] A. Young, "Improving the Direction-Dependent Gain Calibration of Reflector Antenna Radio Telescopes," Ph.D. dissertation, University of Stellenbosch, Stellenbosch, South Africa, 2013.

[22] MatLAB, version 7.8.0.347 (R2009a). Natick, Massachusetts: The MathWorks Inc, 2009.

[23] S. Koziel, S. Ogurtsov, I. Couckuyt and T. Dhaene, "Variable-fidelity electromagnetic simulations and co-Kriging for accurate modeling of antennas," IEEE Trans. Antennas Propag., vol. 61, no. 3, pp. 1301-1308, March 2013.

[24] E. G. Gilbert, "Controllability and observability in multi-variable control systems," SIAM J. Control, vol. 1, no. 2, pp. 128-151, 1963.

[25] FEKO version 7.0. Altair HyperWorks. Stellenbosch, South Africa, 2015. [Online]. Available: www.feko.info

[26] CST Microwave Studio ver. 2015. Computer Simulation Technology. Darmstadt, Germany, 2015. [Online]. Available: www.cst.com

[27] K. Chemmangat, F. Ferranti, T. Dhaene and L. Knockaert, "Tree-based sequential sampling algorithm for scalable macromodeling of high-speed systems," in 16th IEEE Workshop on Signal and Power Integrity (SPI), May 2012, pp. 49-52.

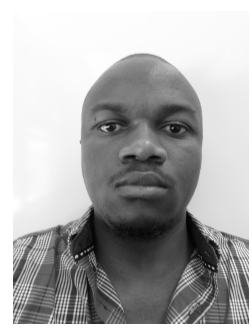

Ngoy Mutonkole received the BEng and MEng degrees in Electrical and Electronic Engineering from the University of Stellenbosch, Stellenbosch, South Africa, in 2011 and 2013 respectively. He is currently working towards the Ph.D. degree at the same university.

His current research interests include antenna design, surrogate based modeling and optimization techniques as well as several aspects of microwave engineering.

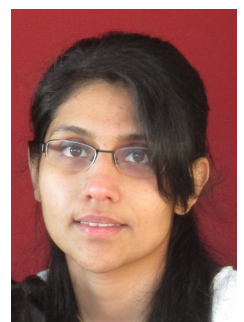

Elizabeth Rita Samuel received the Bachelor of Technology (BTech) degree and the Master of Technology (MTech) degree in Electrical and Electronics Engineering from the University of Kerala, Kerala, India in 2002 and 2005 respectively. She received her Ph.D. degree in Electrical Engineering from Ghent University, Ghent, Belgium in 2015.

Her current research interests include parametric macromodeling and parameterized model order reduction.

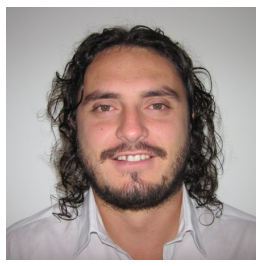

Dirk I. L. de Villiers (S'05-M'08-SM'15) was born in Langbaan, South Africa. He received the B.Eng. and Ph.D. degrees in electrical and electronic engineering from the University of Stellenbosch, Stellenbosch, South Africa, in 2004 and 2007 respectively.

From 2008 to 2009, he was a Post-doctoral Fellow with the University of Stellenbosch working on antenna feeds for the South African SKA program.

$\mathrm{He}$ is currently an Associate Professor at the University of Stellenbosch, and has been a Visiting Researcher with Antwerp University, Antwerp, Belgium, as well as with the University of Novi Sad, Serbia, and Chalmers University of Technology, Gothenburg, Sweden. Between 2010 and 2015, he has worked on contract for EMSS Antennas (Pty) Ltd. in Stellenbosch, South Africa, on the design of the reflector optics for the MeerKAT and SKA radio telescopes. His research interests include reflector antennas as well as the design of antennas and microwave components using surrogate modeling techniques.

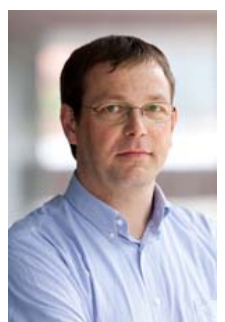

Tom Dhaene (SM'05) received the Ph.D. degree in electrotechnical engineering from Ghent University, Ghent, Belgium, in 1993

From 1989 to 1993, he was a Research Assistant with the Department of Information Technology, Ghent University, where his research focused on different aspects of full-wave electromagnetic circuit modeling, transient simulation, and time-domain characterization of high-frequency and high-speed interconnections.

In 1993, he joined the EDA company Alphabit(now part of Keysight Technologies). He was one of the key developers of the planar EM simulator ADS Momentum. Since 2007, he has been a Full Professor with the Department of Information Technology (INTEC), Ghent University - iMinds. He has authored or co-authored more than 300 peer-reviewed papers and abstracts in international conference proceedings, journals and books. He is the holder of 5 U.S. patents. 